\title{
Laboreal
}

Volume $12 \mathrm{~N}^{\circ} 2$ | 2016

Equipamentos de Proteção Individual II

\section{Para el estudio de la difusión del Modelo Obrero. Tres historias desde Chile}

Para o estudo da difusão do Modelo Operário. Três histórias do Chile

Pour l'étude de la diffusion du Modèle Ouvrier. Trois histoires du Chili

For the study of Workers' Model diffusion. Three stories from Chile

\section{Patrizio Tonelli}

\section{CpenEdition}

Journals

\section{Edición electrónica}

URL: http://journals.openedition.org/laboreal/2181

DOI: 10.4000/laboreal.2181

ISSN: 1646-5237

\section{Editor}

Universidade do Porto

Referencia electrónica

Patrizio Tonelli, «Para el estudio de la difusión del Modelo Obrero. Tres historias desde Chile», Laboreal [En línea], Volume 12 N²2 | 2016, Publicado el 01 diciembre 2016, consultado el 09 octubre 2019. URL : http://journals.openedition.org/laboreal/2181; DOI : 10.4000/laboreal.2181

Este documento fue generado automáticamente el 9 octubre 2019.

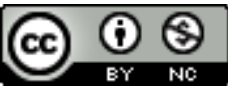

Laboreal está licenciado com uma Licença Creative Commons - Atribuição-NãoComercial 4.0 Internacional. 


\title{
Para el estudio de la difusión del Modelo Obrero. Tres historias desde Chile
}

\author{
Para o estudo da difusão do Modelo Operário. Três histórias do Chile \\ Pour l'étude de la diffusion du Modèle Ouvrier. Trois histoires du Chili \\ For the study of Workers' Model diffusion. Three stories from Chile
}

\section{Patrizio Tonelli}

\section{NOTA DEL EDITOR}

http://dx.doi.org/10.15667/laborealxii0216pt

Manuscrito recebido em: agosto/2016

Aceite após peritagem: outubro/2016

\section{Introducción}

\footnotetext{
“y esta cosa (...) de que todo lo arreglamos con plata (...) porque de repente, solamente, los trabajadores vemos la cosa de las lukas, los sueldos, pero no vemos esta otra cosa, incluso no se negocian cosas así, no se ponen en la negociación colectiva y no es tema" [1].
}

El estudio de experiencias pasadas sirve para revitalizar la acción del presente. En este sentido estudiar cómo en el pasado los trabajadores han abordado la tutela de su salud en el lugar de trabajo nos muestra reflexiones, prácticas y soluciones que pueden ayudarnos a repensar nuestra situación actual y estimular la búsqueda de nuevas estrategias en materia.

El Modelo Obrero representó la "propuesta más acabada" (Villegas \& Ríos Cortázar, 1993, p.63) de investigación participativa en el campo de la salud de los trabajadores, 
haciendo hincapié en los principios de la no-delegación y de la validación consensuada y proponiendo una inédita centralidad de la experiencia de los trabajadores en la generación de conocimiento y acción sobre salud laboral. Su importancia no se quedó limitada al contexto italiano de los años '60 y '70: como Laurent Vogel relata en esta misma revista, a partir de la mitad de los años '70 el Modelo Obrero traspasó los confines italianos para influenciar de distintas maneras, durante las décadas sucesivas, las luchas para la salud de los trabajadores en otros contextos nacionales. Durante los años ochenta, por ejemplo, el Modelo Obrero cruzó el Océano Atlántico arraigándose en América Latina y penetrando la reflexión y la práctica de muchos expertos en salud y dirigentes sindicales.

En los últimos años, una serie de iniciativas nacionales e internacionales han vuelto a estudiar aquella experiencia y su fuerte capacidad de difusión, interrogándose sobre la posibilidad de que el Modelo Obrero Italiano y sus principios sean una fuente de inspiración para las actuales estrategias en salud y seguridad. Se ha tratado de interesantes iniciativas de carácter historiográfico y archivístico $\left.{ }^{2}\right]$ así como de debate entre protagonistas de la época y dirigentes sindicales y expertos activos en la actualidad [ ${ }^{3}$. Las siguientes páginas se inscriben en el marco de estas iniciativas y apuntan a entregar insumos para enriquecer la discusión.

Concretamente, el presente artículo apunta a contribuir al estudio de la difusión del Modelo Obrero en América Latina, y lo hace partiendo de la reconstrucción histórica de tres experiencias acontecidas en Chile durante los años ' 90 del siglo pasado en tres distintos ámbitos laborales: las trabajadoras de pequeños talleres textiles en Santiago, los trabajadores mineros reunidos en la Confederación Minera de Chile, las enfermeras de un hospital público de Santiago.

En esta reconstrucción trataremos de iluminar cómo y porqué los protagonistas de las tres experiencias conocieron el Modelo Obrero, cómo este cambió, adaptándose a contextos y problemáticas nuevas, cómo operó concretamente y qué resultados obtuvo.

Este artículo es el fruto de una investigación llevada a cabo en el marco del programa "Trabajo, Empleo, Equidad y Salud" (TEES) en la Facultad Latinoamericana de Ciencias Sociales (FLACSO), sede Chile, y se ha basado en la recolección de testimonios orales de algunos protagonistas de las tres experiencias relatadas $\left[{ }^{4}\right]$ y de fuentes secundarias de la época.

\section{El encuentro con el Modelo Obrero}

7 La llegada del Modelo Obrero en Chile hunde sus raíces en la segunda mitad de los años '80, cuando al gobierno del país estaba la Junta Militar de Pinochet imponiendo su proyecto de Estado neoliberal. La represión socio-política que siguió el golpe de 1973 y las profundas reformas llevadas a cabo por la dictadura cívico-militar a partir de fines de los años '70 apuntaron a aniquilar el movimiento obrero restituyendo plena soberanía a los intereses empresariales, y a revertir radicalmente las políticas intervencionistas de Estado privatizando las empresas estatales y abriendo los mercados a la competencia global (Salazar \& Pinto, 1999). El shock neoliberal concertado entre los "Chicago boys" [5] y los militares dio lugar a una "obra refundacional de todos los cimientos de la sociedad, de la economía y del Estado" (Gaudichaud, 2015, p.6) que reestructuró profundamente los equilibrios entre capital y trabajo y que impactó significativamente, entre otras cosas, en el ámbito de la salud 
laboral, especialmente en el papel que trabajadores y expertos podían desempeñar y en el espacio de maniobra con que podían contar para la tutela de la salud en los lugares de trabajo.

\subsection{Los expertos conocen el Modelo}

8 Las entrevistadas que en esa época trabajaban como profesionales en el ámbito de la salud laboral recuerdan aquel tiempo por el reducido alcance que caracterizaba la acción de las instituciones públicas y de las disciplinas académicas dedicadas a esos temas.

9 La acción estatal, por un lado, había sido mutilada dado que "prácticamente no quedaba profesional de la salud ocupacional en los servicios (...) porque la dictadura se había encargado de hacer desaparecerlos todos (...)" y los únicos que quedaban eran "unos ingenieros que hacían las autorizaciones sanitarias, y una que otra enfermera y uno que otro médico, pero en la práctica casi nada" [6].

10 Dentro las universidades, por otro lado, las disciplinas vinculadas con la salud de los trabajadores tenían un sesgo que respondía a bien determinados intereses. La psicología del trabajo, por ejemplo, era fuertemente influenciada por "lo que se había hecho en Estados Unidos" y estaba "muy vinculada con cuestiones de eficiencia y de productividad y con psicólogos al servicio de eso".

11 Dos eran, en el fondo, las críticas que algunos sectores de profesionales y académicos movían a ese enfoque dominante: el rol del investigador, "que era el rol del experto que va, que sabe y que dice después lo que hay que hacer" y "una mirada bastante limitada respecto de cuál eran los factores de riesgo de las condiciones de trabajo", reducidos a los factores ambientales [7].

12 La construcción de una visión alternativa que pusiera en discusión los enfoques biomédicos e ingenieriles que dominaban el discurso sobre la salud de los trabajadores se llevaba a cabo en una condición de soledad muy marcada. Relata una joven psicóloga del trabajo de la época que "al inicio fue una búsqueda muy solitaria en Chile. La gente con la cual yo me relacionaba en el área de salud y del trabajo era gente del área médica y del área ingenieril, entonces esta cuestión no estaba presente en el país, esta búsqueda en el debate no se hacía" [8]. La intuición de que otra manera de abordar los temas de salud y trabajo era posible tenía que lidiar con la pesada herencia de una dictadura que había aislado el país, manteniéndolo "totalmente al margen de lo que pasaba, del desarrollo teórico-conceptual $"\left[{ }^{\circ}\right]$.

13 El encuentro esperado, de hecho, fue posible rompiendo los estrechos confines nacionales y viajando a México, a la Universidad Autónoma Metropolitana Xochimilco, donde operaba un grupo de expertos en salud reunidos en torno a la "Maestría en medicina social" que se dictaba en esa universidad. Asa Cristina Laurell y Mariano Noriega, en particular, fueron responsables durante los años '80 de un trabajo de estudio y análisis crítico del Modelo Obrero que tendría importantes efectos en su sucesiva difusión en el continente (Laurell, \& Noriega, 1989; Laurell, 1984). En la opinión de los dos expertos, el Modelo italiano carecía de bases teóricas fuertes al fundar su fortaleza en la sola subjetividad de los trabajadores, lo que hizo depender los resultados del Modelo solo de la situación política concreta en la cual se iba aplicando. Para suplir a este problema, los autores mexicanos proponían integrar en el Modelo una reconceptualización del nexo salud-trabajo que apuntaba a fortalecer un 
"acercamiento global" a los problemas de la salud en el trabajo, y a contrarrestar derivas "ambientalistas" de tipo biomédico (Laurell, \& Noriega, 1989, p.60).

Desde la condición de aislamiento que caracterizaba a los expertos chilenos, el contacto con el Modelo Obrero en México representó un quiebre, la abertura de perspectivas de reflexión y de acción nuevas que sin embargo comunicaban profundamente con sus necesidades de conocimiento e intervención en materia de salud y trabajo y su trayectoria política.

15 Para algunos, por ejemplo, el aspecto más significativo del Modelo Obrero era la especial relación que se generaba entre investigador y trabajadores, en la cual "le preguntan a las personas lo que les pasa. Eso para mí es lo más importante porque si no yo estoy pensando por ti (...)". En las palabras de una enfermera y militante de la época, la posibilidad de romper con un enfoque dominante en el cual el trabajador "no puede opinar, no puede participar, no puede decir lo que le pasa" se conectaba directamente con su trayectoria de educadora popular, construyendo un continuum que permitió el traspaso del modelo nacido en Italia en décadas anteriores a las tierras chilenas [ $\left.{ }^{10}\right]$.

En este sentido se rescataba la valoración operada por el Modelo Obrero del conocimiento como práctica colectiva que nacía a partir de la experiencia de los trabajadores. El saber colectivo "era el reconocimiento de la subjetividad (...) una subjetividad que es memoria, que es saber, que es conocimiento" y los trabajadores "son expertos a modo suyo, su experiencia les da una 'experticidad"'. Rompiendo las tradicionales creencias según las cuales el investigador era el depositario único del conocimiento, en este caso "lo que tú tienes que hacer entonces es ayudar a formalizar, sistematizar ese conocimiento, pero el conocimiento está" [11].

\subsection{Los dirigentes sindicales conocen el Modelo}

17 A diferencia del mundo de los profesionales, para los dirigentes sindicales la necesidad de pensar en un enfoque distinto en materia de salud laboral se alimentaba del escándalo que significaba la realidad diaria de compañeros que morían por accidentes o enfermedades o que estaban sometidos a duras condiciones de trabajo.

Era el caso de los dirigentes de la Confederación Minera de Chile (CONFEMIN), por ejemplo, para los cuales durante los años ' 80 se había transformado en un triste y frecuente ritual juntarse en las zonas productoras de carbón para organizar "una conferencia de prensa en donde denunciábamos la situación producida" por los numerosos accidentes fatales que marcaban al sector y luego participar "al funeral a despedir a nuestros compañeros" (Labraña, n.d., p.12).

En otro sector productivo, aún a comienzos de los años ' 90 , las dirigentas de la Confederación Textil de Chile (CONTEXTIL) tenían que enfrentar el desamparo de las trabajadoras de los pequeños talleres de producción y confección del barrio Patronato [12], que estaban sometidas a pago a trato, jornadas laborales largas, falta de comedores y autoritarismo en el lugar de trabajo que provocaban malestares y enfermedades. “Cómo mierda aguantan tanto? (...) ¿Por qué no se organizaban? ¿Por qué ellas no luchaban?” [13]; “ ¡Hasta cuando nos pasa esto!”. En los dirigentes mineros y textiles estalla a un cierto punto la conciencia de que "actuar así, a permanentes denuncias, era convivir con el actual sistema y dejar las cosas como están" (Labraña, n.d., p.13). El Modelo obrero representó en este contexto una forma de respuesta y un camino para 
encontrar una solución. Para las organizaciones sindicales el contacto con esa experiencia parece pasar por dos canales distintos.

Para la CONFEMIN fue la cooperación sindical internacional que jugó un rol esencial. Para apoyar el fortalecimiento del movimiento sindical chileno recién liberado del peso de la dictadura, a comienzos de los años '90 la central italiana CGIL, a través de su ONG "Progetto Sviluppo", vino a Chile para conversar con la Confederación sobre la posibilidad de hacer un proyecto en materia de salud y seguridad en el trabajo. En la visión de los dirigentes sindicales italianos el proyecto tenía que abarcar ámbitos de intervención distintos como la formación de cuadros, el diseño de un mapa minero de Chile y la legislación nacional en materia de salud laboral. Un aporte central planteado por los italianos era la inclusión del Modelo Obrero en las varias actividades propuestas, "y ahí entendimos que era el camino, nos cayeron como hormas en el zapato" (Labraña, op. Cit., n.d., p.156). El descubrimiento del Modelo Obrero representa un hito que marcará, como veremos más adelante, las prácticas y las reflexiones futuras de la Confederación. Durante los tres años de duración del proyecto, los cursos impartidos por los especialistas italianos contribuyeron a formar un grupo de dirigentes que serán importantes a la hora de difundir entre los trabajadores el principio de no delegación de la salud, pilar del Modelo Obrero [14].

En el caso de las dirigentes de la CONTEXTIL fue esencial el vínculo con algunos profesionales de la Unidad de Salud Ocupacional (USO) del Servicio de Salud Metropolitano Occidente (SSMOcc) [15] que se habían cruzado con las reflexiones y actividades del núcleo de medicina social de la UAM-Xochimilco. Bajo su impulso decidieron utilizar el Modelo Obrero para abordar los graves problemas de salud que las trabajadoras del sector estaban viviendo. La decisión derivaba de la fuerte afinidad percibida con la propuesta, dado que "ese modelo te hace aterrizar (...), a ver tú mismo lo que haces y a descubrir por qué te enfermaste" $\left.{ }^{16}\right]$ y de la necesidad de cambiar en el profundo la mentalidad de las trabajadoras despertando en ellas la conciencia y la necesidad de un cambio de su propia condición.

Entre fines de los años ' 80 y comienzo de los años ' 90 fueron madurando las condiciones para que en distintos sectores productivos algunos profesionales de salud laboral y algunas organizaciones sindicales dieran vida a experiencias de aplicación del Modelo Obrero en Chile, que revisaremos en el próximo capítulo.

\section{Tres experiencias chilenas}

\subsection{El Comedor Acogedor de la Mujer Trabajadora de Patronato}

El elemento más visible de las precarias condiciones laborales en Patronato era que las mujeres no contaban con comedores en los talleres y tenían que consumir sus comidas en la calle, al aire libre, sentadas en la vereda. Por este motivo entre 1993 y 1994, algunas dirigentas de la Confederación Textil decidieron dar vida al Comedor Acogedor de la Mujer Trabajadora del sector Patronato (CODEMU). La iniciativa apuntaba principalmente a crear un espacio "donde ellas venían a almorzar (...) ahí ellas traían su olla, su colación, calentaban y tenían un espacio donde comer y servirse su comida caliente". Paralelamente, para las dirigentas sindicales el Comedor "era un motivo como para llegar a ellas, para organizarlas" [ $\left.{ }^{17}\right]$ creando en sus espacios talleres y actividades de varios 
tipos útiles a las trabajadoras del sector como formación personal, campañas de difusión y fiscalización de los derechos laborales, de sindicalización entre otros [18]. necesariamente que ser de fácil manejo y adaptarse a la experiencia de las participantes. Por eso no fue un producto pensado entre cuatro paredes por algunos especialistas. Así como el Modelo Obrero originario había sido fruto de la interacción e intercambio entre profesionales y trabajadores, la guía del proyecto de CODEMU tenía que pasar por un proceso participativo: "Primero para hacer el manual hicimos varios talleres con ellas e hicimos el manual, después tuvimos el manual que se lo entregamos a ellas y ellas iban trabajando con esto (...)" [23].

Entre tantas actividades de formación y concientización, el tema de la salud laboral ocupaba una posición prioritaria en el Comedor, dados los numerosos problemas de salud que las trabajadoras presentaban por culpa de las malas condiciones en sus lugares de trabajo: problemas a la vista, problemas a las articulaciones, dolores de espalda, estrés. Para abordar esta problemática de forma más contundente las dirigentas de CODEMU, en colaboración con los profesionales del SSMOcc, idearon e implementaron el proyecto "Participación de la mujer trabajadora en la vigilancia y control de la contaminación ambiental en sus lugares de trabajo. Talleres de la confección Patronato, Recoleta", que se llevó a cabo entre octubre 1998 y septiembre 1999 en el sector de Patronato.

La colaboración con los profesionales de la Unidad de Salud Ocupacional del SSMOcc permitió desde un inicio orientar el discurso sobre salud con las traajadoras hacia el Modelo Obrero y sus ejes de fondo: “(...) nosotros les contábamos que en el año tanto allá en Italia también los trabajadores habian visto la misma cosa, y que la cosa de la salud tiene que partir de las personas. Porque de repente tú te preocupai de ganar plata y las lukas y todas esas cosas pero lo otro ni te preocupa entonces... eso te va afectando la salud (...)" [ $\left.{ }^{19}\right]$.

De consecuencia, el proyecto apuntaba prioritariamente $\left[{ }^{20}\right]$ a formar trabajadoras capaces de vigilar y controlar en primera persona las condiciones de salud y trabajo que caracterizaban el proceso productivo, contemplando para eso la organización de cursos para la capacitación de monitoras [21]. En ellos, las trabajadoras de Patronato aprendían a identificar los riesgos y sus causas, a analizar sus efectos " $y$, a partir de su propia realidad (...) elaborar propuestas" [ $\left.{ }^{22}\right]$. En línea con los principios de fondo del Modelo Obrero las dirigentas sindicales del CODEMU así sintetizaban su propuesta: "que los trabajadores seamos también capaces de construir nuestro propio conocimiento sobre la salud laboral y los ambientes de trabajo, con el objetivo de dar solución a los problemas que nos aquejan, en colaboración con profesionales del tema, pero en un trabajo conjunto que nos permita ser protagonistas de los cambios que queremos" (Zapata, \& Pascual, 2000, p.20).

El instrumento principal que tenía que guiar la formación de las monitoras tenía La "Pauta de chequeo de condiciones y medio ambiente de trabajo" que resultó de esta colaboración, de hecho, se hacía cargo de la casi exclusiva presencia de mujeres que trabajaba en los talleres textiles del barrio Patronato insertando la dimensión de género en el análisis de los riesgos para la salud. No era posible obviar el hecho que las participantes al proyecto eran trabajadoras y al mismo tiempo mujeres obligadas a hacerse cargo de sus hogares, sometidas, a diferencia de los hombres, a la doble carga laboral y familiar/domestica. Por eso, el elemento de mayor innovación de la Pauta, respecto del tradicional Modelo Obrero concebido en Italia y de sus integraciones latinoamericanas [ $\left.{ }^{24}\right]$, estaba constituido por la presencia de un sexto grupo de factores 
de riesgo denominado "doble jornada", en el cual considerar el estatus de la trabajadora (estado civil, situación de pareja, aporte al grupo familiar), su grupo familiar (tamaño, presencia de hijos), las tareas domésticas (cuidado de hijos, equipamiento de la casa), las redes de apoyo (acceso a servicios de atención de niños, apoyo para la realización de tareas domésticas), el descanso y recreación (otros empleos remunerados, descanso efectivo), la participación en actividades (participación en actividades sindicales o comunitarias).

Durante los cursos, las futuras monitoras trabajaban en grupos completando de forma progresiva los distintos capítulos de la pauta, que en la primera parte recopilaba y sistematizaba los antecedentes generales del taller (nombre de la empresa, presencia de Comité paritario, número de trabajadores, entre otros) y la descripción del proceso de trabajo (objetos de trabajo, medios de trabajo, secciones, puestos y actividades, diagrama de flujo del proceso para cada producto y croquis de la empresa). En la segunda parte investigaba la presencia de los 6 grupos de riesgo, cada uno de los cuales estaba articulado según distintos agentes de riesgos [ ${ }^{25}$ ]: las trabajadoras tenían que identificar la existencia del riesgo, el número de trabajadores expuestos, y calificar numéricamente la importancia, la probabilidad y la exposición lo que arrojaba un puntaje que permitía clasificar los riesgos en leves, moderados y graves.

31 Al final de los talleres, las monitoras pudieron construir los mapas de riesgo de sus lugares de trabajo, los cuales aportaban antecedentes esenciales para los monitoreos ambientales y el estudio de los puestos de trabajo: "en el manual iban escribiendo, iban poniendo sus cosas, iban haciendo estos planos, porque compramos papel grafito y cartón, tenían los planes de los lugares de trabajo (...)" [26].

\subsection{La Confederación Minera de Chile (CONFEMIN)}

Los tres años de colaboración con la ONG sindical "Progetto sviluppo" impactaron significativamente en las reflexiones y prácticas sobre salud y seguridad de CONFEMIN. Como relata uno de sus dirigentes "estábamos en crisis con todo" [27], incapaces de poner en discusión la visión dominante según la cual la responsabilidad de los accidentes era de los trabajadores y de sus comportamientos riesgosos o que los accidentes eran fatalidades. La acción sindical en materia de salud y seguridad estaba paralizada entre una crítica genérica a la figura del Comité Paritario de Higiene y Seguridad [ ${ }^{28}$, definido como comité "parasitario", y prácticas de delegación y monetarización de la salud.

Reflexionando posteriormente sobre esa época pasada, los dirigentes concluían que el problema arraigaba en la separación que se había naturalizado entre organización sindical y Comité paritario, según la cual cada institución debía transitar por carriles separados y cumplir funciones distintas, lo que implicaba "una deformación de principios que no permite asumir la defensa de la Salud a partir de los trabajadores". Para los representantes de los trabajadores sentados en el Comité, el sindicato se transformaba así en una institución en grado de afectar a su labor, exponiéndolos a tensiones, presiones externas y manejos políticos-partidistas de las cuales había que alejarse o sospechar, confiando al contrario en la visión y en las prácticas de la empresa. Esto significaba, por ejemplo, aceptar que la capacitación del Comité paritario estuviese delegada solo a la Mutual [29] a la cual estaba afiliada la empresa "lo que debilita su accionar como trabajadores. Y por tanto delegan la salud en los empresarios" (Labraña, op. Cit. n.d., p.11). o significaba, por otro lado, pactar en los 
contratos colectivos bonos de seguridad individuales o colectivos que instalaban prácticas perversas en grado de ocultar las responsabilidades de la empresa y descargar sobre los trabajadores los riesgos derivados de condiciones muy peligrosas.

El encuentro con el Modelo Obrero significó comprender que "éramos nosotros que teníamos que elaborar, los trabajadores". De forma organizada los trabajadores tenían ahora que utilizar indistintamente los espacios sindicales o del Comité Paritario, construyendo en ellos un punto de vista autónomo y fuerte sobre los temas de salud y seguridad para ser contraparte frente a los otros actores, cuales el Estado y los empleadores: "el Estado tiene una visión, los empresarios tienen una visión y nosotros tenemos también nuestra visón propia y eso es lo único que nos permite entrar en el escenario para cambiar las cosas" [30].

A partir de esta nueva base conceptual los dirigentes de CONFEMIN elaboraron 2 líneas de acción complementarias que marcaron sus pasos futuros en materia de salud. Se trataba, en primer lugar, de formar nuevos cuadros sindicales en grado de asumir un rol activo en los comités paritarios para luego plantear la participación directa del sindicato en los temas de salud y seguridad.

Concretamente, para CONFEMIN eso se tradujo en la necesidad de construir una figura nueva al interior de la organización, el monitor, a través de la puesta en marcha de programas de formación de monitores, la iniciativa más importante de la Confederación en materia de salud y seguridad.

En la figura del monitor la Confederación condensaba los aprendizajes y los estímulos recibidos por el Modelo Obrero [31]: era una figura interna a la organización sindical en grado de manejar las condiciones de seguridad, salud y del ambiente en el trabajo "desde una perspectiva que nos permita surgir como contraparte". En este sentido el monitor tenía que desarrollar el máximo conocimiento posible de los factores de riesgo existentes en cada empresa y el acceso a la información relacionada [32].

El lugar privilegiado de la acción del monitor era la empresa y en particular el Comité paritario de higiene y seguridad al interior del cual tenían que constituirse como contraparte en grado de disputar la visión de la empresa, creando así las condiciones para que "el comité de parasitario pase a ser a paritario" [ ${ }^{33}$.

El programa de formación de monitores fue un producto que anduvo definiéndose lentamente a partir del comienzo de los años '90 hasta el año 2002, cuando se puso en marcha de forma oficial la Escuela para la formación de los monitores [ ${ }^{34}$. La Escuela tenía una malla curricular estándar que los candidatos monitores debían cumplir en su totalidad. Los cursos se construían en base a 4 niveles que progresivamente iban especializando los contenidos [35], y adoptaban una metodología de enseñanza participativa, basada en actividades orientadas a rescatar la experiencia de los participantes dado que "lo principal son los trabajadores, cómo ellos tienen y han tomado conocimiento" ${ }^{[36]}$.

Uno de los puntos esenciales de la formación de los monitores era aprender, y sucesivamente aplicar en la empresa, el "Modelo Preventivo y Participativo de Mapa de Riesgo". Con este nombre CONFEMIN identificaba las prácticas y orientaciones propias del Modelo Obrero Italiano y de esta forma lo incorporaba en su propio patrimonio de herramientas de acción para construir una estrategia de no-delegación sobre salud y trabajo. 
41 En las elaboraciones de CONFEMIN el modelo de mapa de riesgo tenía que operar pasando por 4 etapas principales, en las cuales encontramos diseminados los varios elementos característicos del Modelo Italiano, tales cómo los grupos homogéneos, la validación consensuada, los cuatro grupos de factores de riesgo.

Para comenzar, el sindicato de empresa y los representantes de los trabajadores del Comité Paritario tenían que juntar a un grupo de trabajadores de una misma sección con los cuales aplicar un ejercicio sobre el cuerpo humano reconociendo y estudiando las principales afecciones que sufrían los trabajadores. Para eso cada trabajador tenía que identificar los principales problemas de salud que le afectaban, los cuales se asociaban a pequeños círculos de papel colorado. Luego, utilizando un papelógrafo sobre el cual se había dibujado un cuerpo humano, los trabajadores involucrados tenían que pegar los papeles en los puntos del cuerpo que correspondían a los problemas detectados. De esta forma el papelógrafo construía un primer mapa que representaba los sufrimientos psicofísicos que afectaban al grupo de trabajadores considerados entregando un registro completo de los problemas más frecuentes y urgentes ${ }^{[37}$. El ejercicio tenía que ser replicado a continuación con otros grupos de trabajadores de la misma sección para obtener, al final, la identificación de las principales afecciones de la sección.

En una segunda etapa el mismo tipo de dinámica se replicaba para mapear los riesgos propios del lugar en el cual el grupo conformado desarrollaba sus labores. En este caso, una vez dibujado el lugar de trabajo en un papelógrafo, con las máquinas y características que lo caracterizaban, el grupo tenía que identificar la condición de aire, las vibraciones, los ruidos, los elementos químicos, los esfuerzos musculares, los movimientos repetitivos de cada trabajador. Cada factor de riesgo se asociaba a otro papel de color que tenía que ser pegado en el papelógrafo, construyendo un mapa de los riesgos identificados y validados colectivamente por el grupo. Junto con eso los trabajadores tenían que asociar los riesgos con los daños a la salud detectados en la primera etapa.

En una tercera etapa, el grupo tenía que discutir sobre los cambios a proponer para eliminar los riesgos. Esta información tenía que ser transmitida al sindicato y a los trabajadores miembros del comité paritario para que tuvieran elementos sobre los cuales basar las negociaciones con la empresa para exigir a la empresa solucionar el problema de los factores de riesgo.

Se iba diseñando de esta forma una estrategia de acumulación de observaciones e informaciones que permitía a la vez "desarrollar una mayor capacidad en cada uno de los trabajadores" y por ende una acumulación de poder por parte de sus representaciones colectivas. Fuerte era la consciencia para CONFEMIN de que el conocimiento acumulado se expresara en una estrategia de negociación: no se trataba simplemente de producir conocimiento científico, había que utilizar ese conocimiento para la acción. Los mapas eran las bases que el comité paritario tenía que dominar "para hacer su plan de seguridad en la empresa" y obligar la empresa a respetarlos [38].

Central en esta dinámica era considerar el Modelo preventivo y participativo de mapa de riesgo como el fruto de una construcción horizontal: el éxito de la iniciativa dependía necesariamente de la participación activa "de todos los trabajadores en su proceso de elaboración y construcción. Ya que por este camino se produce un aprendizaje masivo" (Labraña, op. Cit. n.d., p.93). 


\subsection{Las enfermeras del hospital Sotero del Río}

El tercer ejemplo considerado relata una experiencia diferente respecto de las anteriores. En este caso el Modelo Obrero no fue aplicado en un contexto de acción sindical si no que como metodología de estudio cualitativo en un proyecto de investigación en psicología laboral. En el año 1994 un grupo de investigadoras postuló a los fondos nacionales para la investigación científica, Fondecyt [ $\left.{ }^{39}\right]$, con el proyecto "La salud mental ocupacional de las enfermeras del sub-sector público de salud en Chile". Las impulsoras del proyecto, una psicóloga y una enfermera, se habían conocido en la UAM Xochimilco estudiando con el núcleo de medicina social de esa universidad y, fascinadas por el potencial crítico y heurístico del Modelo obrero, decidieron utilizarlo para estudiar la salud psicológica de las enfermeras de un hospital público de Santiago, el hospital Sotero del Río.

Los objetivos del proyecto apuntaban de forma prioritaria a reconocer los riesgos para la salud mental derivados de las condiciones de trabajo de las enfermeras, a "diseñar una estrategia preventiva de autocuidado de las enfermeras" y a contribuir "a la toma de decisiones en materia de salud laboral a nivel de los Servicios de Salud, de las autoridades del Sector y del propio gremio de las enfermeras" (Avendaño, Grau, \& Yus, 1999, p.16). Consecuentemente se planificaron dos años de tiempo para la recolección y análisis de datos, a través de métodos cualitativos y cuantitativos, y un tercer año para la formulación de propuestas.

El primer año del proyecto, dedicado al estudio cualitativo, vio como protagonista al Modelo Obrero que sin embargo fue adaptado respecto de su versión italiana y mexicana, para ser aplicado en el sector de los servicios a la persona, contexto muy diferente del sector industrial en el cual había sido generado. Además las investigadoras evaluaban que el Modelo, pensado para un universo masculino, no tenía en cuenta la realidad de trabajadoras dependientes obligadas también a hacerse cargo del trabajo doméstico, como eran las enfermeras; en último sentían la exigencia de adecuar el Modelo al estudio de factores de riesgo para la salud psicológica presentes en el las condiciones de trabajo que, según ellas, estaban poco representados en el Modelo originario. En particular, las reflexiones y los cambios concernieron la identificación de los grupos de los factores de riesgo y la construcción de los grupos homogéneos.

Respecto del primer punto, el resultado de las adaptaciones fue la "creación de un grupo de riesgos sobre condiciones de trabajo doméstico que se agregó a la versión mexicana" (Avendaño, 1996, p.30). Como en el caso de Patronato analizado anteriormente, el Modelo Obrero aplicado con las enfermeras del Sotero del Rio también se amplió al estudio y discusión de seis factores de riesgo.

51 En el caso de la identificación de los grupos homogéneos las investigadoras se enfrentaban al reto de adaptar el criterio de homogeneidad, que implicaba reconocer a aquellos grupos que vivían los mismos riesgos, a la realidad de un hospital: “Qué era lo que entendíamos con compartir los mismos riesgos? (...) Si uno se quedaba pegado en el modelo inicial de interpretación de riesgos era casi el puesto de trabajo, y nosotros pensábamos que no era así porque (...) los riesgos de la organización y división del trabajo no son los de tu puesto de trabajo, tienen que ver con la administración de la empresa entonces ahí hay un tema (...)" [40]. Las reflexiones llevaron finalmente a utilizar la complejidad en la atención de enfermería, "que es el criterio que se usa para clasificar las camas" [11], para identificar a cuatro grupos homogéneos: las enfermeras que trabajan en unidades de alto riesgo 
(Urgencias y Unidades de Cuidado Intensivo); las enfermeras clínicas o tratantes que trabajan en medicina y especialidades con pacientes de mediana dificultad; las enfermeras supervisoras, con tareas de menor complejidad; las enfermeras de atención primaria.

Las participantes del proyecto fueron seleccionadas de forma voluntaria gracias a la intermediación del Colegio de enfermeras, organización gremial del sector, con la cual las investigadoras tenían fuertes vínculos [42].

La primera parte del trabajo de los grupos homogéneos estuvo dedicada a reconstruir el proceso de trabajo de las enfermeras, para lo cual se habían creado distintas actividades orientadas a estimular un trabajo participativo de las mismas [ $\left.{ }^{43}\right]$. Terminada la reconstrucción del proceso productivo, las siguientes sesiones estaban dedicadas al trabajo con la Encuesta Colectiva, instrumento que ordenaba las percepciones del grupo sobre los riesgos y que consistía de una tabla que en su columna vertical ordenaba los distintos riesgos laborales a identificar (los seis grupos de factores de riesgo), y en su parte horizontal identificaba cómo y dónde se manifestaban, su impacto y propuestas de medidas de prevención.

Durante las discusiones, el protagonismo otorgado a la experiencia de las mismas trabajadoras permitía develar problemas o riesgos que una mirada externa de profesionales no imaginaba deber tener en cuenta: “¿el ruido es un problema, es un riesgo para la salud de las enfermeras? Entonces de repente empezaban unas: "sí claro porque los cabros chicos gritan, porque las mamás gritan" o sea no es un riesgo a la sordedad profesional, sino es el otro ruido... y así fueron saliendo miles y miles y miles de cosas" [44].

La capacidad de las discusiones colectivas de las enfermeras de iluminar aspectos no considerados en fase de diseño de la intervención fue particularmente valorada por parte de las responsables del proyecto y las obligó a cambiar sobre la marcha la manera de trabajar con el instrumento de Encuesta Colectiva o directamente a modificar algunas convicciones teóricas adquiridas durante sus años de estudio. Importante, por ejemplo, se demostró integrar la recolección de los disensos que se producían dentro del grupo "cuando eran relevantes o significativos, porque también proporcionaban información" respecto de percepciones, dinámicas, decisiones en materia salud. En otro caso, las investigadoras tuvieron que revisar la idea, contenida en la Encuesta y fundamentada por la literatura científica de la época, de que la polifuncionalidad constituyera un riesgo: "Nos encontramos que no y por más que indagué los grupos al respecto, para las enfermeras la gran diversidad de tareas que asumían no era un riesgo si no que era un beneficio, enriquecía su tarea" y por eso no era vivida como un riesgo "si no que por el contrario un protector" $\left.{ }^{45}\right]$.

56 Al final del ciclo de sesiones cada grupo sintetizó el proceso vivido de socialización y reapropiación de la información acerca de los riesgos contenidos en el proceso de trabajo, construyendo mapas de riesgo.

Dado que en la Encuesta Colectiva había un espacio dedicado a proponer medidas preventivas para combatir los riesgos, el trabajo con el Modelo Obrero proporcionó también insumos clave para elaborar las propuestas para el mejoramiento de las condiciones de vida y de trabajo de las enfermeras, que correspondían al tercer y último año de investigación. Aquí, la riqueza del trabajo de los grupos homogéneos desarrollado durante el primer año volvió a manifestarse en la amplitud de las propuestas hechas por las mismas enfermeras, las cuales abordaron "cuestiones muy concretas sobre cómo reorganizar el trabajo, (...) cuestiones desde el estilo de liderazgo, los 
sistemas de recompensa (...)" [ $\left.{ }^{46}\right]$ y fueron estructuradas en cuatro niveles: el nivel de las políticas públicas, el nivel de acción en los establecimientos, el nivel de acción gremial y el nivel personal.

58 La presencia de propuestas que abordaban desde el autocuidado individual hasta el cuestionamiento de la organización del trabajo (cambio en los sistemas de turnos, redefinición del rol de las enfermeras y de la enfermería en los servicios, entre otros) confirmó para las responsables del proyecto las potencialidades inscritas en el Modelo obrero, como metodología para la investigación y para la acción política transformadora. La conexión generada durante los grupos homogéneos entre la condición individual y colectiva de las trabajadoras, de hecho, permitió que la crítica y las exigencias individuales asumieran inmediatamente un carácter político que planteaba otra manera de organizar la sociedad y las relaciones de poder. "Las propuestas que formulamos parten de una concepción de sociedad que hoy día no existe y que es necesario construir. Una sociedad justa, equitativa y solidaria. En que se respetan y garantizan los derechos de todos sus ciudadanos a la salud y al trabajo (...) una sociedad que dignifica el trabajo y lo humaniza" (Avendaño, 1996).

\section{Resultados y conclusiones finales}

Las experiencias relatadas permiten comenzar a reconstruir uno de los muchos caminos tomados por el Modelo Obrero Italiano fuera de su contexto originario. En este capítulo final se apunta a resumir los principales resultados de las experiencias, identificando la existencia de ámbitos problemáticos o de factores que estimularon su implementación. Para eso se seguirán algunas de las reflexiones que caracterizan el renovado interés hacia el Modelo Obrero a nivel internacional: la importancia de la experiencia subjetiva de los trabajadores, la redefinición de los roles entre sujeto y objeto en el proceso de investigación y aprendizaje, el significado de la no-delegación [47]. De esta forma se espera rescatar insumos valiosos para el debate sobre propuestas de acción crítica y transformadora en temas de salud y seguridad.

Un resultado que los tres casos comparten tiene relación con la posibilidad ofrecida por el Modelo Obrero de movilizar la experiencia subjetiva de los trabajadores involucrados estimulando procesos de toma de conciencia colectiva. En este sentido el trabajo con el Modelo Obrero se caracteriza por ser "un proceso de aprendizaje" en el cual los trabajadores van "haciendo propios los hallazgos" $\left.{ }^{48}\right]$ y de esta forma van comprendiendo el proceso productivo de forma integral hasta "visualizar (...) que lo que vivían y como lo vivían era no tan individual, era colectivo y tenía que ver con la naturaleza del trabajo, sus condiciones de trabajo (...) fue un gran cambio" [ $\left.{ }^{49}\right]$.

61 El momento fundamental en que se generaba el aprendizaje era el grupo homogéneo, el proceso de discusión y validación consensuada sobre riesgos y proceso productivo que ahí se daba, ya que en ese espacio los trabajadores "negocian percepciones acerca de esa realidad y construyen una mirada que es colectiva y que deja de ser "yo tengo problemas con la jefa", o "tengo problemas con el médico" o "a mí no me pagan bien" [50].

62 La reapropiación de una visión colectiva sobre su propia realidad es un evento tan radical que para las trabajadoras de Patronato significó literalmente "abrir los ojos". Antes del contacto con el Modelo Obrero "había gente que llegaba al lugar de trabajo y se sentaba en su puesto y no veía nada más, no veía sus compañeros y la forma en que ellos trabajaban ni que ellas trabajaban porque ellas no se daban ni cuenta"; durante los talleres, el 
desarrollo de la Encuesta Colectiva, las discusiones en grupo "se empezaron a dar cuenta, como a mirar más porque no se habían dado el tiempo (...) como verse de las condiciones en que ellas trabajaban". Absorbidas en los ritmos frenéticos impuestos por las necesidades de vivir y de trabajar "no tenían esa forma de hacer la reflexión, el cómo yo estoy trabajando", y aceptaban como naturales las condiciones en las cuales estaban insertadas. Fue en el espacio de los grupos, estudiando y conociendo los puestos de trabajo, que aprendieron a tomar distancia crítica de su condición, poniendo en discusión y en ridículo esa supuesta naturalidad, y descubriendo que era justamente esa la causa de su malestar [51].

63 La toma de conciencia no se limitó al lugar de trabajo sino que desveló los nocivos efectos que el trabajo irradiaba sobre la vida entera de las trabajadoras. Eran por ejemplo las relaciones de pareja a ser miradas de forma distinta: para las enfermeras por ejemplo "apareció mucho el asunto de ser mujer sola, porque era muy generalizado o sea la cantidad de mujeres que tenían una relación de pareja "estable", saludable, sana, era bajísima y de repente se van dando cuenta de que eso tiene que ver con el trabajo y el cómo trabajan y en qué trabajan" [ ${ }^{52}$. La condición de mujer también, con la doble carga de trabajo que la sociedad le asignaba, comenzó a tener una consideración crítica. Muchas de las cosas que hasta ese minuto las trabajadoras textiles habían hecho de forma natural y necesaria, como preocuparse de planificar la jornada de los hijos y las comidas de la familia, eran vistas ahora como problemas que afectaban a su calidad de vida, como una "sobrecarga (...) que hacía de que ellas se fueran enfermando" [53].

Un factor que caracterizaba el trabajo al interior del grupo homogéneo y que facilitó el proceso de reapropiación descrito fue el tipo de papel que jugaron los profesionales de salud en el acompañar a los trabajadores durante las intervenciones, el cual hacía referencia a principios que eran propios también de la educación popular [54]. Diversamente de los modelos tradicionales de intervención social, estas propuestas coincidían en plantear que "el conocimiento lo descubren las personas" dado que era un producto de su experiencia directa. En materia de salud este punto de vista sostenía que "ellos saben que la gente se enferma, por qué se enferma, donde están los riesgos", y el experto (médico, psicólogo, ergónomo, enfermera, etc.) tenía que asumir el rol de “ facilitador" [55]. Concretamente, la Encuesta Colectiva de Riesgos aplicada en el proyecto de Patronato era concebida como instrumento de educación popular.

De las experiencias chilenas emerge, entonces, que uno de los factores que impulsaron la difusión del Modelo Obrero en el continente se relaciona con su capacidad de establecer una conexión con prácticas y corrientes teóricas ya existentes y activas en América Latina, como la educación popular de Paulo Freire (1970), la Investigación acción participativa (Fals Borda, 1987) o la medicina social latinoamericana (Waitzkin, Iriart, Estrada, \& Lamadrid, 2001).

En esta misma línea, otro factor que facilitó su difusión fue su flexibilidad y capacidad de adaptarse a condiciones distintas de las originales. En contacto con nuevos contextos prácticos y nuevos estímulos teóricos, las personas que utilizaron el Modelo Obrero fueron capaces de reformular sus herramientas de acción aun respetando sus principios de fondo sobre el protagonismo de la experiencia de los trabajadores (validación consensual y no-delegación). Los casos de las enfermeras del Sotero del Rio y de las trabajadoras textiles de Patronato nos han mostrado, por ejemplo, la posibilidad de reformular los grupos homogéneos y los grupos de factores de riesgo para una realidad distinta de la grande fábrica taylorista poblada por obreros manuales varones. La 
creación de un sexto grupo de factores de riesgo sobre "doble jornada" de la mujer trabajadora constituía a este propósito una contribución notable para abrir los temas de la salud y del trabajo a las temáticas de género. Finalmente, el Modelo demuestra no ser un dogma estático.

No obstante todo lo anterior, la reconstrucción llevada a cabo en esta investigación describe experiencias limitadas e incapaces de poner en marcha acciones colectivas que modificaran el panorama sindical y laboral chileno de la época.

Una razón de fondo de estos muy débiles éxitos reside en el contexto de relaciones laborales autoritarias vigentes en Chile a partir de 1973 y sobre todo del año 1979, cuando el "Plan Laboral" instaló un marco regulatorio que apuntó a despotenciar e inhibir sistemáticamente la acción colectiva de los trabajadores (Narbona, 2015). Por eso ninguna de las experiencias relatadas tuvo resultados muy contundentes a nivel de movilización o de negociación colectiva; sin embargo es posible registrar en dos de ellas (CODEMU y CONFEMIN) algunos logros significativos.

69 En el caso de los pequeños talleres textiles de Patronato las dirigentas del CODEMU destacan algunos resultados concretos que mejoraron un poco la calidad de vida de las trabajadoras como la instalación de comedores en los lugares de trabajo y de Comités paritarios de higiene y seguridad. Junto con eso, un cambio significativo tuvo que ver con la distinta actitud que las mismas trabajadoras asumieron frente a los temas de salud laboral. "Ellas empezaron a descubrir que (...) cuando se reunían con el Comité paritario (...) podían hacer actas y depositarlas a la Inspección del trabajo (...) que antes no se hizo". Una mayor conciencia de sus propias razones y de los medios a través de los cuales hacerlas valer parece ser así un fruto importante en términos de prácticas de hegemonía, entendiendo con eso la capacidad de incidir más allá de su específico contexto laboral para forzar a las instituciones existentes a cambiar, adecuarse al nuevo escenario y ampliar su propia función a favor de los trabajadores. En la Dirección del Trabajo, por ejemplo, "se hizo un departamento de salud laboral (...)" y se estimuló el desarrollo de estudios en materia de salud y condiciones laborales de las trabajadoras de otros sectores. Está claro para las dirigentes del CODEMU que estos cambios fueron posible gracias a una nueva proactividad de las mismas trabajadoras "porque si nosotros a la Inspección del trabajo no demandamos sobre estas cosas no hacen nada". Otro fruto de esta nueva actitud fue la creación al interior de la Dirección del Trabajo de un equipo operativo dedicado a fiscalizaciones especiales durante las veinticuatro horas del día [56].

70 En la misma línea de acumulación de conciencia y de construcción/utilización de instrumentos de poder, podemos registrar la conformación de nuevos sindicatos. Junto con el nacimiento de cuatro sindicatos de empresa, las trabajadoras del sector conformaron un sindicato interempresa ${ }^{[57}$ para organizar a esos contextos laborales que no contaban con los mínimos legales establecidos para formar a un sindicato de empresa.

71 La eficacia de estas dinámicas de cambio de actitud y de los equilibrios de poder se refleja finalmente en la reacción del orden constituido, que en varias ocasiones se dedicó a amenazar y amedrentar a las responsables del CODEMU. La acción conjunta de los patrones de los talleres y de la fuerza pública apuntaba justamente a impedir o poner trabas a un tipo de experiencia que lentamente estaba rompiendo con una situación que tradicionalmente había aceptado como naturales o inmodificables los 
daños a la salud de las trabajadoras y que ahora estaba contribuyendo a concientizarlas y movilizarlas [58].

En el caso de CONFEMIN también es posible registrar algunos efectos concretos y cambios en las prácticas en materia de salud y trabajo. Es el caso del Contrato colectivo de trabajo de la mina Quebrada Blanca que indicaba una contra tendencia respecto de las normales prácticas de "monetarización" presentes en el sector. En vez de reivindicar bonos o indemnizaciones, el sindicato comprometía la empresa "a tomar todas las medidas necesarias para proteger eficazmente la vida y la salud de los trabajadores manteniendo las condiciones adecuadas de higiene y seguridad en faena en Iquique como también los implementos necesarios para prevenir accidentes y enfermedades profesionales (...). La compañía y el sindicato realizarán reuniones regulares en forma trimestral para tratar las materias relacionadas con la salud, la seguridad y el medioambiente".

En la misma línea los dirigentes de la Confederación citan a los acontecimientos del mineral subterráneo "El Indio", donde como sindicato "habíamos dicho que si habían lugares inseguros se cerraba (...) va transitando un trabajador por el lugar (...) se desprende un planchón y lo mató: entonces los 3 sindicatos, porque eran 3 sindicatos que formaban el "Consejo El Indio", se fueron inmediatamente al paro, y comenzó a haber una reacción en que participa el sindicato. Esto era parte de una cultura preventiva (...)" [59].

El proyecto de las enfermeras, por otro lado, no tuvo resultados concretos en términos de negociación colectiva, organización o lucha de los trabajadores. Las propuestas que las investigadoras habían sistematizado junto a las enfermeras durante los grupos homogéneos "fueron entregadas al Colegio de enfermeras (...) les pedimos que las publicaran, $y$ nunca la publicaron": el fruto más concreto del proyecto no encontró el respaldo de la organización ni influyó en su patrimonio de consciencia y prácticas en materia de salud y trabajo. Utilizando las palabras de una de la responsable del proyecto, ese trabajo “ cayó en tierra de nadie". La ausencia de resultados prácticos tiene relación con los objetivos de ese proyecto y el rol de la organización de trabajadores en su planteamiento: "Fue una experiencia que nace por la iniciativa de algunas investigadoras que la aplican para una investigación: el fin de ella no era la movilización de los trabajadores, más bien la investigación" [ $\left.{ }^{60}\right]$.

De este ejemplo se desprende que otra razón de la débil o nula presencia de resultados concretos de estas intervenciones reside en el tipo de relación que estas iniciativas mantienen con la organización de los trabajadores. En aquellos casos donde el sindicato es protagonista de la intervención, siendo su inspirador y contribuyendo a diseñarlo y desarrollarlo, es posible reconocer una cierta capacidad de incidir en la realidad, aun débil. Si la organización de los trabajadores no juega ese rol los resultados prácticos son nulos.

Desde un inicio, por ejemplo, el proyecto realizado por el CODEMU en Patronato fue una experiencia orientada a la investigación-acción, entendiendo con eso una práctica cuyos objetivos fundamentales consisten en "la generación de conocimiento, la difusión y socialización de ese saber generado y la movilización organizada en torno a las condiciones nocivas de trabajo y salud" (Noriega \& Villegas, 1993, p. 11). En esa experiencia "estaba involucrada una federación de distintas organizaciones sindicales que estaban haciendo un proyecto ambiental y ahí nos invitaron" [ ${ }^{61}$. El Comedor Acogedor de la Mujer Trabajadora de Patronato (CODEMU), organización nacida al alero de la CONTEXTIL, había construido una agenda propia de temas y actividades, dentro de la cual la salud cobraba una importancia especial. En este contexto la organización tenía 
un rol preponderante y activo en el diseño de la intervención, en la elaboración de los materiales, en el desarrollo de las actividades y en el seguimiento. Lo mismo se puede decir de la experiencia de CONFEMIN.

Aparece aquí entonces la centralidad de la no delegación, pilar de fondo del modelo obrero, y de las elecciones que implica: ¿Quién tiene el protagonismo, los trabajadores o los investigadores? ¿Hasta dónde se hacen cargo los dirigentes sindicales? ¿Cuánto delegan a los investigadores? Preguntas complejas y que implican un constante trabajo de revisión crítica, dado que hasta las experiencias aparentemente más positivas esconden dificultades en el gestionar el difícil confín que separa la delegación de la nodelegación.

En el caso de CODEMU, por ejemplo, las dirigentas señalaban como un punto crítico la manera en que ellas habían gestionado la relación entre profesionales y organización sindical. Reconocían haber tenido "muchas expectativas hacia el trabajo que podía realizar el profesional (...) por tanto se descansa en él". La muy criticada delegación en materia de investigación y de decisiones en materia de salud terminó presentándose nuevamente en la "mucha dependencia de USO para llevar a cabo muchas actividades" o en el escaso involucramiento que las dirigentas tuvieron "en la discusión metodológica del proyecto, por ejemplo el Modelo Obrero Italiano" (CODEMU, n.d., p.1). Se devela de estos apuntes la reiteración de patrones de intervención en salud que, si en teoría eran fuertemente criticados, en la práctica terminaban por caracterizar profundamente las acciones y los proyectos emprendidos.

Las tres experiencias reconstruidas lanzan entonces el desafío de profundizar en esta contradicción a la hora de ampliar la mirada sobre la difusión del Modelo Obrero en América Latina, dado que muchos casos podrían presentar una relación "bastante parcial " con el Modelo Obrero. En ellos "se recupera la idea del mapa de riesgo pero no se recupera el Modelo Obrero italiano en su integridad", reduciendo el Modelo a una mecánica y estéril producción de mapas: "se trata de construir un mapa de riesgo pero el proceso mismo que para mí es lo enriquecedor, lo que hace que se provoque el cambio en la gente creo que se desdibujó de alguna otra experiencia por lo que he visto. Muchos publican mapas de riesgos pero no sé si en esta perspectiva (...) de investigación-acción" [62].

\section{BIBLIOGRAFÍA}

Alhaique, D. (2011). L'archivio del Centro Ricerche e Documentazione dei rischi e danni da lavoro (CRD): un progetto di recupero. In O. Bianchi y G. Chianese (Eds.), Lavoro, salute, sicurezza. Uno sguardo lungo un secolo, Annali della Fondazione Di Vittorio 2010, Roma, Ediesse.

Avendaño, C. (1996). Salud mental Ocupacional de las enfermeras del subsector público de salud chileno, Proyecto Fondecyt n 1940752, Archivo Nacional Chile, CONICYT, P. Fondecyt, Carpeta 990. Santiago: Chile.

Avendaño, C., Grau, P., \& Yus, P. (1999). Riesgos para la salud de las enfermeras del sector público en Chile, Revista Colegio de Enfermeras, año 30 ${ }^{\circ}$, n. 102, 15-26. 
CODEMU (1999). Conociendo nuestro ambiente de trabajo: protejamos nuestra salud. Informe de evaluación. Condiciones y medioambiente de trabajo, Pauta de chequeo. Santiago: Chile.

CODEMU (n.d.). Evaluación del primer proyecto FDLA por parte del equipo CODEMU. Mimeo. Santiago: Chile.

Fals-Borda, O. (1987). The application of participatory action-research in Latin America, International sociology, vol. 2, $n^{\circ} 4,329-347$.

Freire, P. (1970). Pedagogía del Oprimido. Montevideo, Tierra Nueva.

Gaudichaud, F. (2015). Las fisuras del neoliberalismo maduro chileno: trabajo, "democracia protegida" y conflictos de clases. Buenos Aires. CLACSO. Extraido en agosto, 2, 2016 de: http://

biblioteca.clacso.edu.ar/clacso/becas/20150306041124/EnsayoVF.pdf

Harvey, D. (2007). Breve historia del neoliberalismo. Madrid, Akal.

Labraña, M. (n.d.). Algunas opiniones sobre el tema de la seguridad. Mimeo. Santiago: Chile.

Laurell, A. C. (1984). Ciencia y experiencia obrera: la lucha por la salud en Italia, Cuadernos políticos, n. 41, 63-83.

Laurell, A. C., \& Noriega, M. (1988). La salud de los trabajadores siderúrgicos y la reconversión industrial, Salud Problema, 15, 9-34.

Laurell, A. C., \& Noriega, M. (1989). La salud en la fábrica. Estudio sobre la industria siderúrgica en México. México D.F.: Ediciones Era.

Ministerio de Salud (1992). Planificación estratégica. Desarrollo de la salud ocupacional en Chile a 5 años. Santiago de Chile.

Narbona, K. (2015). Para una historia del tiempo presente: lo que cambió el Plan Laboral de la dictadura, Fundación SOL, Ideas para el buen vivir, $\mathrm{n}^{\circ} 6$.

NESst (2002). Corporación Comedor Acogedor de la Mujer Trabajadora /CODEMU: Gestionando un negocio no relacionado a la misión, Serie de Casos NESsT, n. 6, Chile, de: http://www.gestionsocial.org/ archivos/00000505/NESST-CODEMU-2002.pdf

Noriega M., \& Villegas J. (1993). La investigación participante en la salud laboral. Avances y limitaciones a 10 años de distancia. Salud Problema, 23, 11-19.

Salazar G., \& Pinto, J. (Ed.) (1999), Historia contemporánea de Chile. Vol. II. Actores, identidad y movimiento Santiago. Santiago: LOM.

Servicio de Salud Metropolitano Occidente - Unidad Salud Ocupacional, CODEMU, Consejo de las Américas (1999). Participación de la mujer trabajadora en la vigilancia y control de la contaminación ambiental en sus lugares de trabajo. Talleres de la confección Patronato Recoleta. Región Metropolitana. Resumen ejecutivo de resultados del proyecto. Santiago: Chile.

Villegas Rodríguez, J., \& Ríos Cortázar, V. (1993). La investigación participativa en la salud laboral: el modelo obrero. In A. C. Laurell (Ed.), Para la investigación sobre la salud de los trabajadores, (pp. 63-98), Serie PALTEX, Salud y Sociedad 2000, n. 3, Organización Panamericana de la Salud.

Waitzkin, H., Iriart, C., Estrada, A., \& Lamadrid, S. (2001). Social medicine then and now: lessons from Latin America, American Journal of Public Health, 91, 10, 1592-1601.

Zapata, C., \& Pascual, C. (2000). Guía sindical de salud laboral y ambiente de trabajo. Comedor Acogedor de la Mujer Trabajadora de Patronato (CODEMU), Central Unitaria de Trabajadores (CUT). Santiago de Chile. 


\section{NOTAS}

1. Dirigenta sindical. Entrevista. 22 junio 2013.

2. En Italia el INAIL (el instituto nacional para la compensación de accidentes laborales) y las tres centrales sindicales italianas están llevando a cabo un proyecto de digitalización y publicación de los archivos del Centro de Investigación y Documentación sobre riesgos y daños del trabajo (CRD), que fue, entre 1974 y 1984, el Centro sindical unitario encargado de recopilar y difundir las experiencias de luchas obreras y sindicales en contra de la nocividad. El archivo contiene casi 7000 documentos producidos entre 1961 y 1983 por delegados sindicales, comisiones de fábrica, sindicatos, expertos involucrados en la lucha para la salud de los trabajadores (Alhaique, 2011).

3. Se hace referencia específicamente al seminario desarrollado por el Instituto de los Sindicatos Europeos (ETUI) en Bruselas en febrero del año 2016: http://www.etui.org/Topics/Health-Safety/ News/The-struggle-for-health-at-work-the-Italian-workers-model-of-the-1970s-as-a-source-ofinspiration

4. Fueron entrevistados: una dirigente sindical del sector textil, un dirigente sindical del sector minero y dos investigadoras.

5. Con esta expresión se identifica un grupo de economistas chilenos que entre años '50 y '60 perfeccionaron sus estudios en la Universidad de Chicago, bajo la guía del economista neoliberal Milton Friedman. Durante la dictadura de Pinochet (1973-1990) fueron los responsables de la restructuración de la economía chilena a través de la aplicación de políticas que redujeron el gasto fiscal, privatizaron la seguridad social, permitieron la explotación privada y desregulada de los recursos, facilitaron la inversión extranjera directa y una mayor libertad de comercio (Harvey, 2007).

6. Investigadora. Entrevista. 28 mayo 2013. El diagnóstico estaba compartido por el mismo Ministerio de Salud que en el año 1992 lanzaba los lineamientos estratégicos para el desarrollo de la salud ocupacional en Chile hasta 1997: "Recursos Humanos: Enfrentados a un Servicio de salud con un recurso humano insuficiente para atender la demanda creciente de la población, se hace necesario ir aumentando en forma paulatina su número, su capacitación técnica y ejecutiva" (Ministerio de Salud, 1992, p. 5).

7. Investigadora. Entrevista. 14 octubre 2013.

8. Íbid.

9. Investigadora. Entrevista. 28 mayo 2013.

10. "Yo ya venía también del mundo de la educación popular entonces entenderás que este modelo a una la convocaba absolutamente, eso para mí era lo más obvio, porque estaba pegado a la tierra, porque expresaba lo que las personas vivíamos". Ibid.

11. "Uno de los aspectos del Modelo que me alucinó fue esta idea del saber colectivo. Eso fue para mí así como... de un impacto. Cuando leí en un artículo de Salud Problema que había escrito Cristina pero así que me abrió el mundo y las posibilidades o sea. Hoy puedo decir que en términos epistemológicos fue un quiebre, un antes y un después pero diría que en ese minuto fue lo más que me impactó junto con esto de la no-delegación en salud, la no-monetarización del riesgo. Pero el saber colectivo fue lo que me marcó". Investigadora. Entrevista. 14 octubre 2013.

12. El barrio de Patronato “(...) se encuentra ubicado en un sector antiguo de Santiago, físicamente en la ribera norte del rio Mapocho, es un sector comercial y fabril, caracterizado por su alta concentración de micro, pequeñas y medianas empresas de la industria del vestuario y en menor grado, empresas de la industria textil". (Servicio de Salud Metropolitano Occidente Unidad Salud Ocupacional, CODEMU \& Consejo de las Américas, 1999, p. 7).

13. Dirigenta sindical. Entrevista. 22 junio 2013.

14. Para el año 1992, por ejemplo, durante 12 días de formación "formamos 20 dirigentes sindicales en cursos más intensivos con el Proyecto Sviluppo sobre la Seguridad, Salud e impacto 
del Ambiente en las Condiciones de Trabajo y en el Principio de la no delegación de la Salud por parte de los trabajadores" (Labraña, n.d., p. 158).

15. En aquella época, antes de la reforma de 2004, las Unidades de Salud Ocupacional (USO) eran parte de los Servicios de Salud territoriales y dependientes del Ministerio de Salud de Chile.

16. Dirigenta sindical. Entrevista. 22 junio 2013.

17. Ibid.

18. Para una profundización de las actividades de CODEMU, así como de sus formas de gestión y de sus cambios en el tiempo ver: NESst (2002). Corporación Comedor Acogedor de la Mujer Trabajadora /CODEMU: Gestionando un negocio no relacionado a la misión, Serie de Casos NESsT, n. 6, Chile, disponible en: http://www.gestionsocial.org/archivos/00000505/NESSTCODEMU-2002.pdf.

19. Dirigenta sindical. Entrevista. 22 junio 2013.

20. Paralelamente, se contemplaban otros objetivos y actividades complementarias: - la medición del impacto de las condiciones medioambientales de trabajo en salud a través del examen de salud laboral preventivo de 140 trabajadoras de los talleres y el monitoreo ambiental "de los 15 talleres participantes al proyecto"; - la difusión de estas temáticas en el territorio con la constitución de un "Comité de acción para la defensa del medio ambiente". (Servicio de Salud Metropolitano Occidente - Unidad Salud Ocupacional (et al.), 1999, p. 9-10).

21. "La convocatoria a las trabajadoras para participar se realizó a través de invitaciones personalizadas que se les hizo llegar a los talleres con los cuales se habían realizado alguna actividades previamente". (Servicio de Salud Metropolitano Occidente - Unidad Salud Ocupacional (et al.), 1999, p. 13).

22. El Curso-taller contempló 24 horas pedagógicas, impartido a dos grupos de trabajadores (as). En total se capacitaron 27 personas (Zapata \& Pascual, 2000, p. 9).

23. Dirigenta sindical. Entrevista. 22 junio 2013.

24. En América Latina se había agregado un quinto grupo de factores de riesgo: los riesgos derivados de las instalaciones, máquinas y equipos. (Laurell \& Noriega, 1988).

25. El I Grupo estaba constituido por 7 agentes (temperatura, humedad, ventilación, ruido, etc.); el grupo II por 8 agentes (polvos, gases, hongos, bacterias, etc.); el grupo III por 2 agentes (Actividad física intensa, posiciones incómodas); el IV grupo por 10 agentes (jornada de trabajo, tipo de remuneración, ritmo, monotonía, trabajo fragmentado, supervisión estricta, etc.); el V grupo por 5 agentes (Edificios, Instalaciones, maquinarias, herramientas, etc.); el VI grupo por 21 ítems (hijos pequeños a cargo, composición núcleo familiar, etc.). (Servicio de Salud Metropolitano Occidente - Unidad Salud Ocupacional (et al.), 1999, p. 14-15). Ver también: CODEMU, 1999.

26. Dirigenta sindical. Entrevista. 22 junio 2013.

27. Dirigente sindical. Entrevista. 4 Junio 2013.

28. El Comité Paritario de Higiene y Seguridad (CPHS) es el organismo técnico de participación entre empresas y trabajadores, para detectar y evaluar los riesgos de accidentes y enfermedades profesionales. Según la Ley 16.744 los Comités Paritarios son obligatorios en empresas con más de 25 trabajadores y deben estar integrados por tres representantes de la empresa y tres de los trabajadores.

29. Las Mutuales (o Mutualidades de Empleadores) son corporaciones de derecho privado, sin fines de lucro, creadas por los empleadores entre el año 1957 y 1966. Tienen por objeto administrar el seguro social contra riesgos de accidentes del trabajo y enfermedades profesionales, según lo establecido por la Ley 16.744. En Chile existen actualmente 3 Mutualidades de Empleadores: Instituto de Seguridad del Trabajador (IST), Asociación Chilena de Seguridad (ACHS), Mutual de Seguridad (MUSEG).

30. Dirigente sindical. Entrevista. 4 Junio 2013. 
31. "Nosotros dijimos que había que formar monitores. Viene de la parte del modelo obrero: los monitores son dirigentes sindicales, miembros de comités paritarios, son miembros elegidos por el sindicato, el sindicato tiene que elegir las personas que tienen el perfil para hacer el curso para monitores" (Labraña, n.d., p. 16).

32. "La no delegación implica que tanto el sindicato, nuestra parte del Comité Paritario y el conjunto de los trabajadores deben tomar conocimiento en la empresa de cada uno de los incidentes, accidentes, factores de riesgos, agentes contaminantes químicos y nocivos ambientales, metodologías de medición de las tasas de accidentabilidad, etc.". Ibid., p. 18.

33. Dirigente sindical. Entrevista. 4 Junio 2013.

34. Durante el primer curso, que se desarrolló en septiembre 2002 durante 16 días por un total de 120 horas pedagógicas, se formaron a los primeros 30 monitores (Labraña, n.d.).

35. En el primer (nivel A) los participantes recibían formación sobre el marco legal vigente en materia de salud y seguridad en el trabajo, con especial énfasis también sobre las elaboraciones de OIT. En el nivel B se abordaba el Decreto no 72 que establecía el "Reglamento de Seguridad Minera". En el nivel C se estudiaban los impactos de los tóxicos en el trabajo. El nivel D se dedicaba a profundizar en la metodología participativa de construcción de Mapa de riesgos (Labraña, n.d.).

36. Dirigente sindical. Entrevista. 4 Junio 2013.

37. “Una vez que se ha trabajado con el grupo de la sección. Se evalúa toda la información. Si las personas con las que se trabajó fueron 8 por decir un ejemplo, y se evalúan 6 dolores de cabeza ocasionales - 4 malestares al estómago - 5 malestares a la espalda - 6 tendinitis - etc. Toda esta información se guarda con los nombres de los evaluados" (Labraña, n.d., p. 89).

38. "Se entra en la etapa de negociación partiendo del hecho que el mapa de riesgo siempre va a ser su lectura la estrategia que va a tener que dominar el comité paritario para hacer su plan de seguridad en la empresa, anual, entonces habrán planes anuales, planes a corto plazo, a mediano plazo y a más largo plazo, entonces aquello que la empresa se compromete a resolver en tanto tiempo en seis meses, en un año, en dos años". Dirigente sindical. Entrevista. 4 Junio 2013.

39. "El Fondo Nacional de Desarrollo Científico y Tecnológico, Fondecyt, tiene por objetivo estimular y promover el desarrollo de investigación científica y tecnológica básica, y es el principal fondo de este tipo en el país". http://www.conicyt.cl/fondecyt/sobre-fondecyt/que-esfondecyt/

40. ] Investigadora. Entrevista. 28 mayo 2013.

41. "(...) en el proceso de atención de enfermería tú te das cuenta que la gravedad de los pacientes sí constituye un riesgo". Investigadora. Entrevista. 28 mayo 2013.

42. En total participaron 36 enfermeras, con un promedio de 9 por grupo. Investigadora. Entrevista. 28 mayo 2013.

43. Se contemplaba por ejemplo la visión de un diaporama que representaba una jornada típica de una enfermera desde que se levantaba hasta que se acostaba; o el trabajo con un listado de "todas las actividades que hacíamos las enfermeras (...) para que ellas pudiesen reflexionar sobre la carga de trabajo, qué significaba todo el trabajo que hacíamos". Investigadora. Entrevista. 28 mayo 2013.

44. Investigadora. Entrevista. 28 mayo 2013.

45. Investigadora. Entrevista. 14 octubre 2013.

46. Ibid.

47. Sobre esas reflexiones ver, por ejemplo, las presentaciones realizadas durante el seminario de febrero 2016 en Bruselas, disponibles en: http://www.etui.org/Topics/Health-Safety/News/Thestruggle-for-health-at-work-the-Italian-workers-model-of-the-1970s-as-a-source-of-inspiration

48. Investigadora. Entrevista. 28 mayo 2013.

49. Investigadora. Entrevista. 14 octubre 2013.

50. Ibid. 
51. "Después ellas se reían de las sillas, porque las sillas estaban llenas de cototos y cojines, entonces ellas no se daban cuenta de esas cosas". Dirigenta sindical. Entrevista. 22 junio 2013.

52. Investigadora. Entrevista. 14 octubre 2013.

53. "Entonces ahí se ve que la sobrecarga que tienen una parte es la cosa psicológica porque estai haciendo el trabajo y estai pensando, no es que tu estí ahí en la máquina, "métele no más", sino que tu mente por otro lado también está funcionando entonces todo eso era una sobrecarga de trabajo para ellas y que hacía de que ellas se fueran enfermando". Dirigenta sindical. Entrevista. 22 junio 2013.

54. El Modelo Obrero "es lo mismo que hacía el Paulo Freire, es muy parecido, es lo más parecido y por eso me hizo mucho sentido" Investigadora. Entrevista. 28 mayo 2013.

55. "Los viejos saben todo, la diferencia está en que desde los profesional uno se lo va a traducir en una norma técnica, lo va a traducir en otra cosa, pero los viejos tienen el conocimiento (...) entonces por eso me hizo tanto sentido a mí a diferencia de otros modelos, donde hay la variable tanto me dice que la regresión no sé qué, lo que me dice no sé qué, y que hay un riesgo relativo de 2.1... eso a mí no me dice mucho". Ibid.

56. Dirigenta sindical. Entrevista. 22 junio 2013.

57. El sindicato interempresa alcanzó a tener entre 250 y 270 mujeres afiliadas. Ibid. Según el Código del Trabajo chileno para constituir un sindicato de empresa se requiere un mínimo de 25 trabajadores: los trabajadores de aquellas empresas que no cumplen con dicho quorum pueden conformar un sindicato Interempresa que, según la misma ley, es un sindicato que agrupa trabajadores pertenecientes a dos o más empleadores distintos. El quorum para la conformación de un sindicato interempresas también es de 25 trabajadores (esta vez de empresas distintas).

58. "Igual no fue fácil (...) una vez llegó el equipo operativo de carabineros con metralleta y todas esas cosas al lugar. Y nos habían acusado que nosotros teníamos un casino clandestino que ellos venían a buscar eso (...) Incluso nos seguían: cuando nosotras nos veníamos de Patronato no podíamos andar solas". Ibid.

59. Dirigente sindical. Entrevista. 4 Junio 2013.

60. Investigadora. Entrevista. 28 mayo 2013.

61. Ibid.

62. Investigadora. Entrevista. 14 octubre 2013.

\section{RESÚMENES}

El Modelo Obrero representó durante los años setenta la "propuesta más acabada" de investigación participativa en el campo de la salud de los trabajadores y desde su lugar de origen, Italia, se difundió influenció las luchas para la tutela de la salud de los trabajadores de otros contextos nacionales. El presente artículo presenta una reconstrucción histórica de tres experiencias de aplicación del Modelo Obrero en Chile durante los años '90 del siglo pasado en tres distintos ámbitos laborales. En ellas se destacan la capacidad del Modelo de adaptarse a contextos y exigencias nuevas así como los problemas ligados a la práctica de la no-delegación. El artículo pretende ser un aporte historiográfico que enriquezca el estudio y comprensión del Modelo y de sus fortunas pasadas, apuntando, al mismo tiempo, a redescubrirlo como aporte para repensar las actuales formas en que trabajadores y expertos abordan la salud laboral.

O Modelo Operário representou durante os anos setenta a "proposta mais acabada" de investigação participativa no campo da saúde dos trabalhadores, e a partir de seu local de origem, a Itália, se difundiu e influenciou as lutas pela proteção da saúde dos trabalhadores em outros contextos nacionais. Este artigo apresenta uma reconstrução histórica de três experiências de 
aplicação do Modelo Operário no Chile durante os anos '90 do século passado, em três diferentes ambientes de trabalho. Nelas se destacam a capacidade do Modelo de se adaptar a contextos e exigências novas, assim como os problemas ligados à prática de não delegação. $\mathrm{O}$ artigo pretende ser um aporte historiográfico que enriqueça o estudo e a compreensão do Modelo e de suas riquezas passadas, se direcionando, ao mesmo tempo, a redescobri-lo como aporte para repensar as formas atuais com que trabalhadores e especialistas abordam a saúde no trabalho.

Le Modèle Ouvrier a représenté au cours des années soixante-dix la proposition plus complète de recherche participative dans le domaine de la santé des travailleurs et, depuis son milieu d'origine l'Italie, a influencé la lutte pour la protection de la santé des travailleurs dans d'autres contextes nationaux. Cet article présente une reconstitution historique de trois expériences d'application du Modèle Ouvrier au Chili pendant les années 90 dans trois domaines de travail différents. Ces expériences montrent la capacité du modèle à s'adapter à de nouveaux contextes et exigences, de même qu'aux problèmes liés à la pratique de la non-délégation. Cet article prétend être une contribution historiographique pour enrichir l'étude et la compréhension du Modèle Ouvrier et de ses fortunes passées, et tentant au par-là de le redécouvrir en tant que contribution à repenser les façons actuelles dans lesquelles les travailleurs et les experts traitent la santé au travail.

During the seventies, the Italian Workers' Model represented the "most complete" participatory action research (PAR) proposal in the field of workers' health. From Italy, it spread and influenced the struggle for the protection of the workers' health of other national contexts. This article presents a historical reconstruction of three experiences in implementing the Italian Workers' Model in Chile during the 90s of the last century in three different working places (sectors). These experiences show, on the one hand, the ability of the model to adapt to new contexts and demands and, on the other hand, the problems linked to the practice of nondelegation. The paper intends to be an historiographical contribution to enrich the study and understanding of the Italian Workers' Model and its past success, pointing at the same time, to rediscover it as a contribution to rethink the current ways in which workers and experts address the occupational health domain.

\section{ÍNDICE}

Mots-clés: histoire, santé des travailleurs, Chili, modèle ouvrier

Palabras claves: historia, salud de trabajadores, chile, modelo obrero

Keywords: history, health at work, Chile, Italian Worker's Model

Palavras-chave: história, saúde dos trabalhadores, chile, modelo operário

\section{AUTOR}

\section{PATRIZIO TONELLI}

Programa Trabajo, Empleo, Equidad y Salud (TEES), Facultad Latinoamericana de Ciencias Sociales (FLACSO) - sede Chile. Dirección: Terranova 248/F, Providencia, Santiago, Chile. patriziotonelli@flacsochile.org. 